\title{
Linx
}

Revue des linguistes de l'université Paris X Nanterre

39 | 1998

Modèles linguistiques : convergences, divergences

\section{Des journées entières dans les arbres, et après ?}

\section{Karine Baschung}

\section{OpenEdition}

Journals

Édition électronique

URL : http://journals.openedition.org/linx/862

DOI : $10.4000 / \operatorname{lin} x .862$

ISSN : 2118-9692

Éditeur

Presses universitaires de Paris Nanterre

\section{Édition imprimée}

Date de publication : 15 décembre 1998

Pagination : 7-11

ISSN : 0246-8743

\section{Référence électronique}

Karine Baschung, «Des journées entières dans les arbres, et après ? », Linx [En ligne], 39 | 1998, mis en ligne le 26 juin 2012, consulté le 22 septembre 2020. URL : http://journals.openedition.org/linx/862 ; DOI : https://doi.org/10.4000/linx.862

Ce document a été généré automatiquement le 22 septembre 2020.

Département de Sciences du langage, Université Paris Ouest 


\title{
Des journées entières dans les arbres, et après?
}

\author{
Karine Baschung
}

1 Ce numéro de LINX intitulé "Modèles linguistiques: convergences, divergences " se veut une contribution à certaines tentatives récentes ${ }^{1}$ de dresser un bilan épistémologique de la théorie linguistique contemporaine. En l'occurrence -- et le pluriel est l'essence de la discussion -- il est évidemment nécessaire de parler des théories linguistiques contemporaines.

\section{Convergences autour d'un « paradigme post- chomskyen »}

2 A l'intérieur de ce qu'il est désormais convenu d'appeler le paradigme post-chomskyen, admettons en effet -- sans reprendre en détail les discussions y relatives -- qu'ont émergé les points de consensus suivants :

3 (i) un modèle représentationnel monostratal, i.e. non dérivationnel, est supérieur à un modèle bi- ou multi-stratal (sur la faillite de la grammaire générative transformationnelle, cf. entre autres Milner (1989)) ;

4 (ii) les grammaires syntagmatiques «modernes» (Borsley, 1996) sont supérieures aux grammaires syntagmatiques classiques et à leurs représentations en arbres de constituants ;

5 (iii) les modèles "multi-niveaux" spécifient plus adéquatement que les modèles classiques l'interaction simultanée ou parallèle entre les différentes composantes linguistiques (phonologie, morphologie, lexique, syntaxe, sémantique, voire pragmatique) ;

6 (iv) en vertu du principe de "parcimonie ontologique ", les modèles "surfacistes » sont supérieurs aux modèles qui postulent des entités abstraites non observables (e.g. des catégories vides). 
7 Des exemples de démonstration à cet égard se trouvent aussi bien du côté des grammaires dites d'unification ou basées sur les contraintes (LFG, GPSG, Grammaires Catégorielles, HPSG, TAG) que par exemple dans la Word Grammar de Hudson (1984), la Syntaxe Autolexicale de Sadock (1991), ou la Grammaire d'Arbres Polychromes de Cori \& Marandin (1993). Des progrès théoriques et empiriques sont ainsi attestables entre autres dans le traitement sans transformations des phrases passives ou impersonnelles, dans le traitement sans effacement des phrases ou des constituants coordonnés, ou encore dans le traitement sans catégories vides des phrases à extraction.

\section{Divergences...}

8 Mais, comme en témoigne la prolifération elle-même des modèles actuels, il subsiste une absence évidente de consensus sur la description et la théorisation de nombreux phénomènes linguistiques :

9 (i) un modèle doit-il postuler ou non la notion de (structures de) constituants en syntaxe? On sait que la position négative est défendue par la Grammaire Cognitive (Langacker, 1987), mais également par les Grammaires Catégorielles ou par exemple par le même Hudson (1984) ;

10 (ii) un modèle doit-il ou non remettre en cause plus radicalement encore la partition classique entre règles vs. entrées lexicales?;

11 (iii) un modèle doit-il s'inscrire ou non dans la lignée du programme génératif de Chomsky (1957) ? On peut certes opposer aux tenants de la Grammaire Cognitive la nécessité épistémologique de la contrainte de décidabilité, mais la question demeure de savoir si, hors d'un paradigme cognitif précis, toutes les options descriptives (les «littéralisations» au sens de Milner (1989)) ne peuvent pas être considérées comme équivalentes ;

12 (iv) pour tout modèle complexe se pose le problème de l'évaluation du compromis entre pouvoir formel («Qui peut le moins peut le plus») et contenu empirique (sa « couverture » descriptive). Plus le degré de libéralisme analytique est élevé, moins le système semble devenir prédictif : jusqu'à quel point alors tout ne peut-il pas être décrit, voire décrit de plusieurs manières? Comment identifier ce qui ne serait que variantes notationnelles?

13 Autant de questions qui demeurent largement ouvertes. Certes la multiplicité théorique est normalement inhérente à la discipline linguistique comme à d'autres. Les modèles ne sont pas a priori équivalents: en tant qu'ils sont une instance d'observation, ils ne découpent pas et ne hiérarchisent pas de la même manière les données empiriques. Reste à interpréter leur manque actuel de stabilisation comme signe de vitalité et/ou d'immaturité.

\section{Traitement automatique de la langue}

14 A noter que le programme génératif pose lui-même question aujourd'hui au traitement automatique de la langue. Indépendamment du constat qu'on ne sait pas très bien ce qu'un analyseur -- par exemple -- est censé tester ou valider relativement à une théorie linguistique (cf. Marandin (1993)), on peut se demander si l'homogénéisation soi-disant constatable actuellement représente réellement autre chose pour les divers 
modèles que le fait de manger consciemment ou inconsciemment aux principaux râteliers théoriques et formels en vogue. Mais il est significatif à cet égard que le rapport final d'EAGLES sur les formalismes linguistiques implémentables (EAGLES, 1996) ait conclu -- malgré certains points de convergence indéniables entre les formalismes LFG, TAG et HPSG -- à l'impossibilité de définir un standard de représentation (un métalangage) unique et/ou commun. Si le métalangage est par définition compatible avec une variété d'analyses, et si c'est la théorie qui contraint celles-ci, on se départira provisoirement, par conséquent, d'un optimisme unificateur à la Abeillé \& Blache (1997) dans leur état de l'art sur la syntaxe.

Si la grammaire est un algorithme, i.e. un automate abstrait qui permet, à partir d'un ensemble fini de règles, d'engendrer ou de décrire l'infinité des phrases de la langue, la contrainte de décidabilité est questionnée par le TAL dans la mesure où la notion de phrase grammaticale semble elle-même remise en cause par les travaux sur grands corpus (cf. Karlsson et al. (eds) (1995); AfLA (1996)). En outre, presque tous les formalismes grammaticaux contemporains ont la capacité générative d'une machine de Turing, i.e. leur pouvoir formel est équivalent à des grammaires de type 0 dans la hiérarchie de Chomsky-Schützenberger. Il est clair que les exigences linguistiques sur les formalismes courants sont incompatibles pour le moment avec celles de la recherche et du développement en linguistique-informatique.

Par ailleurs, le problème de l'évaluation de l'équivalence éventuelle des descriptions reste entier. Pour reprendre inter alia l'exemple de la Constraint Grammar de Karlsson et al. (eds) (1995), il est probable -- de l'aveu de ses auteurs mêmes -- que ses axiomes soient redondants, sans qu'il existe actuellement un moyen clair d'en calculer la redondance.

Autant de questions qui demeurent largement ouvertes donc, et qui ne prétendent bien sûr pas être résolues ici. Pour une tentative de clarification, du moins les contributions proposées dans ce volume tentent-elles de mettre en avant, en même temps que la présentation de recherches en cours, une réflexion d'ordre épistémologique au croisement de modèles linguistiques différents.

\section{BIBLIOGRAPHIE}

ABEILLÉ, A. (1993) Les nouvelles syntaxes ĐĐÊGrammaires d'unification et analyse du fran ‘ais, Colin, Paris.

ABEILLÉ, A. \& GODARD, D. (eds) (1996) Nouveaux raisonnements syntaxiques, Langages $n^{\circ} 122$, Larousse, Paris.

ABEILLÉ, A. \& BLACHE, P. (1997) « Etat de l'art : la syntaxe », TAL vol. 38 n², pp. 69-90, ATALA, Klincksieck, Paris.

AFLA (1996) « Corpus : problèmes de constitution et d'exploitation », Revue franaise de linguistique appliquŽe vol. 1-2, Association Française de Linguistique Appliquée, Paris. 
BASCHUNG, K. (1992) Grammaires d'unification ^ traits et contr ${ }^{T M} l e$ des infinitives en franais, Adosa, Clermont-Ferrand.

BORSLEY, R. (1996) Modern Phrase Structure Grammar, Basil Blackwell, Oxford.

BOUQUET, S. (ed) (1998) DiversitŽ de la (des) science(s) du langage aujourd'hui ĐĐ Figures, modles et concepts ŽpistŽmologiques, Langages $\mathrm{n}^{\circ} 129$, Larousse, Paris.

CHOMSKY, N. (1957) Structures syntaxiques, trad. française 1969, Le Seuil, Paris.

CORI, M. \& MARANDIN, J.-M. (1993) « Grammaires d'arbres polychromes », TAL vol. 34 n 1, pp. 101-132, ATALA, Klincksieck, Paris.

EAGLES (1996) « Formalisms Working Group -- Final Report », Expert Advisory Group on Language Engineering Standards, C.E.E. Project LRE-61-100, http: // www.ilc.pi.cnr.it / EAGLES / home.html.

HUDSON, R. (1984) Word Grammar, Basil Blackwell, Oxford.

KARLSSON, F., VOUTILAINEN, A., AIKKILÄ, J. \& ANTILLA, A. (eds) (1995) Constraint Grammar: A Language-independent System for Parsing Unrestricted Text, Mouton de Gruyter, Berlin.

LANGACKER, R.W. (1987) Foundations of Cognitive Grammar ĐĐ vol. I Theoretical Prerequisites, Stanford University Press, Stanford.

MARANDIN, J.-M. (1993) « Analyseurs syntaxiques -- Equivoques et problèmes », TAL vol. 34 n 1, pp. 5-33, ATALA, Klincksieck, Paris.

MILNER, J.-C. (1989) Introduction ^une science du langage, Le Seuil, Paris.

SADOCK, J.M. (1991) Autolexical Syntax: a Theory of Parallel Grammatical Representations, The University of Chicago Press, Chicago.

\section{NOTES}

1. Cf. Langages $\mathrm{n}^{\circ} 122, \mathrm{n}^{\circ} 129$.

\section{AUTEUR}

\section{KARINE BASCHUNG}

Université Paris X Nanterre

UFR LLPHI

Département des Sciences du Langage

GRIL - Groupe de Recherche dans les Industries de la Langue

Université Blaise Pascal Clermont II

Formation Doctorale Linguistique \& Informatique 